\title{
Esofagitis necrosante aguda: análisis retrospectivo
}

\author{
R. Ramos, J. Mascarenhas, P. Duarte, C. Vicente y C. Casteleiro \\ Servicio de Aparato Digestivo. Hospital Universitário de Covilhã. Covilhã, Portugal
}

\section{RESUMEN}

Introducción: la esofagitis necrosante aguda es una entidad rara. Se reconoce por el aspecto negro difuso del esófago a la endoscopia. Su incidencia e patogénesis se desconoce.

Pacientes y métodos: se analizaron retrospectivamente 11 pacientes con esofagitis necrosante aguda desde el punto de vista de los datos clínicos, de laboratorio y endoscopicos en 2 años.

Resultados: se analizaron las endoscopias realizadas a 3.976 pacientes, observándose esofagitis necrosante aguda en 11 pacientes. El estado nutricional era malo en 6 pacientes. La resolución completa de la esofagitis se observó en cuatro pacientes. Durante el seguimiento se observó una estenosis en un paciente y un nuevo episodio de esofagitis necrosante aguda en otro paciente. Siete pacientes fallecieron, pero esta elevada mortalidad parece deberse a las enfermedades de base y no es atribuible a las lesiones de la esofagitis necrosante.

Conclusiones: la incidencia de esofagitis necrosante aguda en nuestra serie fue $0,28 \%$. La esofagitis necrosante aguda tiene una elevada mortalidad.

Palabras clave: Esofagitis necrosante aguda. Necrosis de la mucosa. Endoscopia.

\begin{abstract}
Background: acute esophageal necrosis has been considered a rare event. It is defined as the presence of diffuse dark pigmentation of the esophagus on upper endoscopy. Its incidence has not yet been established. The pathogenesis remains unknown.

Patients and methods: a retrospective analysis of clinical, laboratory, endoscopic, and histological data, and of the clinical course of 11 patients with acute necrotizing esophagitis was carried out over a 2-year period.

Results: among 3,976 patients who underwent upper endoscopy, $11(0.28 \%)$ with acute esophageal necrosis were identified. Nutritional status was poor for 6 patients. Complete resolution of acute esophageal necrosis without further recurrence was observed in 4. One stricture appeared during follow-up and other patient developed new-onset acute esophageal necrosis. Seven patients died, but no death was directly related to acute esophageal necrosis.

Conclusions: the incidence of acute esophageal necrosis in our series is $0.28 \%$. Acute esophageal necrosis is associated with high mortality rates.
\end{abstract}

Key words: Acute esophageal necrosis. Mucosal necrosis. Endoscopy.

Ramos R, Mascarenhas J, Duarte P, Vicente C, Casteleiro C. Esofagitis necrosante aguda: análisis retrospectivo. Rev Esp Enferm Dig 2008; 100: 583-585.

\section{INTRODUCCIÓN}

La esofagitis necrosante aguda (ENA) es una entidad de descripción muy infrecuente. Se reconoce por el aspecto negro difuso del esófago a la endoscopia, en aso-

Recibido: 14-01-08.

Aceptado: 21-04-08.

Correspondencia: Rui Ramos. Servicio de Aparato Digestivo. Hospital Universitário de Covilhã. Quinta do Alvito. 6000-251 Covilhã, Portugal. email: ruimmramos@gmail.com ciación a cambios histopatológicos de necrosis de la mucosa. La necrosis es mayor en el tercio distal con terminación abrupta en la línea $\mathrm{Z}$.

La primera descripción endoscópica fue informada por Goldenberg y cols. (1) en 1990. La causa de la lesión no está completamente aclarada, aunque en ella parece intervenir un componente isquémico (1); obstrucción al tracto de salida gástrico (2); hipersensibilidad a antibióticos (3) e infecciones virales (4). El pronóstico es variable. En muchos casos se asoció a un curso fatal, aunque en otros casos no es un evento terminal. Esto sugiere que el pronóstico depende de la edad y de las enfermedades de base. 
En el presente estudio se analizaron 11 casos de ENA diagnosticados en una Unidad de Endoscopia durante 24 meses. El objetivo de nuestro trabajo fue detectar la frecuencia, patogénesis, evolución clínica y pronóstico.

\section{PACIENTES Y MÉTODO}

Entre marzo de 2005 y abril de 2007, 11 pacientes (edad media de 76,2 años) presentaron ENA. Se realizó un estudio descriptivo en los pacientes con necrosis esofágica realizando un análisis retrospectivo de las características endoscópicas, hallazgos histológicos y tratamiento. Igualmente se analizaron los aspectos patogénicos, clínicos, paraclínicos (pruebas de laboratorio) y evolutivos de estos pacientes. El estado nutricional general fue evaluado determinando las proteínas totales y albúmina en la sangre.

Los criterios diagnósticos de ENA incluyen presentación aguda; hallazgos endoscópicos de un esófago negro con terminación abrupta en la unión gastroesofágica (Fig. 1: paciente $\mathrm{n}^{\circ} 5$ ); se deben excluir otras causas de esofagitis aguda (tóxica, traumática, cáusticos o agentes virales); hallazgos histológicos con compromiso de la mucosa y submucosa (Fig. 2: paciente $\mathrm{n}^{\circ}$ 5).

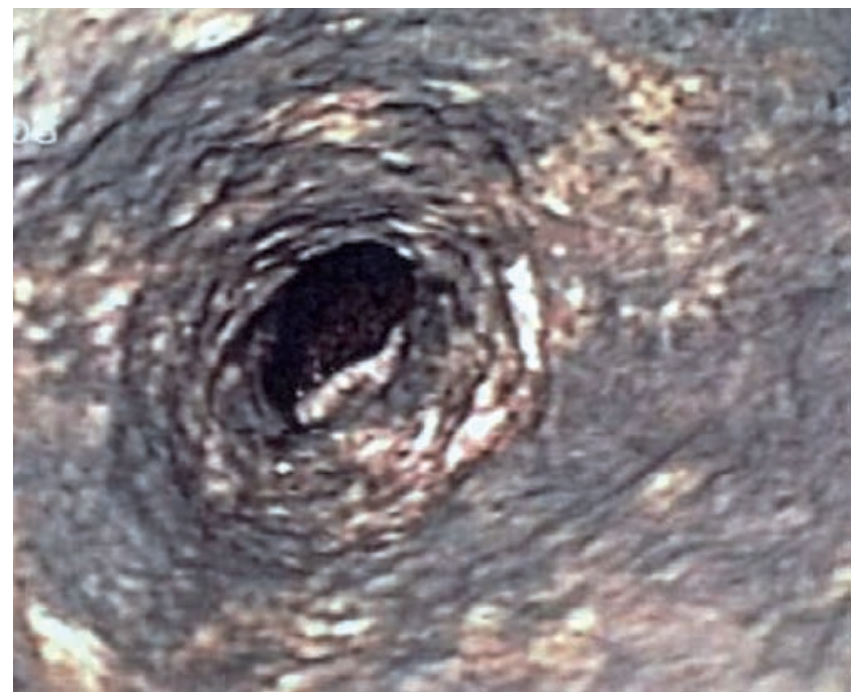

Fig. 1. Aspecto negro difuso del esófago (paciente $n^{\circ} 5$ ).

\section{RESULTADOS}

En una Unidad de Endoscopia se realizaron 3.976 gastroscopias en 24 meses (marzo 2005 y abril 2007) y se estableció el diagnóstico de ENA en 11 pacientes $(0,28 \%), 9$ varones y 2 mujeres. La manifestación inicial fue deposiciones melénicas y/o hematemesis, o vómitos "en poso de café". Un paciente presentó dolor abdominal (Tabla I).

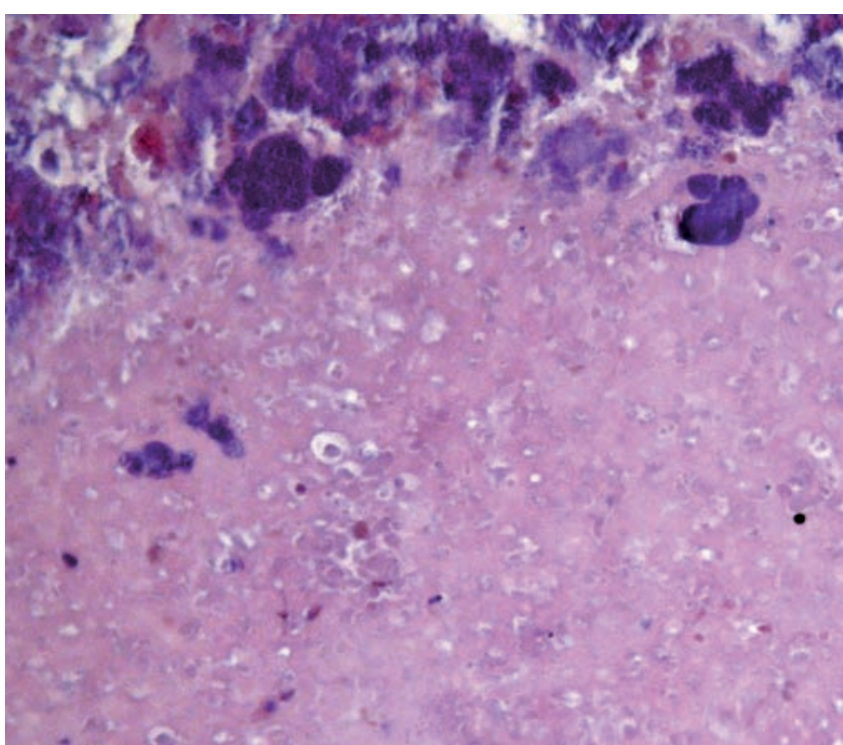

Fig. 2. Necrosis de la mucosa y submucosa (paciente $n^{\circ} 5$ ).

Tabla I. Características clínicas de los pacientes con esofagitis necrosante aguda

\begin{tabular}{lll}
\hline Paciente $n^{\circ}$ & Edad/sexo & Síntomas \\
\hline 1 & 68/mujer & Melena y hematemesis \\
2 & 87/varón & Melena \\
3 & 69/varón & Hematemesis \\
4 & $71 /$ varón & Vómito "en poso de café" \\
5 & 78/varón & Melena y hematemesis \\
6 & 74/mujer & Dolor abdominal \\
7 & 83/varón & Vómito "en poso de café" \\
8 & 76/varón & Melena y hematemesis \\
9 & 65/varón & Vómito "en poso de café" \\
10 & 83/varón & Hematemesis \\
11 & 84/varón & Melena y hematemesis \\
\hline
\end{tabular}

Nueve pacientes presentaron insuficiencia cardiaca. Dos pacientes tenían antecedentes de cirrosis hepática y tres mostraron infecciones. Dos de los pacientes presentaron ENA tras intervenciones quirúrgicas. Un paciente tenía antecedentes de trauma grave. Dos de los pacientes presentaron insuficiencia renal y deshidratación grave. El estado nutricional era malo en 6 pacientes. Cuatro pacientes presentaron anemia (Tabla II).

Tabla II. Aspectos clínicos y enfermedades de base de los 11 pacientes

\begin{tabular}{lcl}
\hline Enfermedades & $(n)$ & (casos) \\
\hline Insuficiencia cardiaca & 9 & $1,3,4,5,6,7,9,10,11$ \\
Cirrosis & 2 & 2,8 \\
Infecciones & 3 & $2,6,7$ \\
Hipoproteinemia & 6 & $2,5,6,8,9,11$ \\
Tras intervenciones quirúrgicas & 2 & 6,7 \\
Trauma & 1 & 10 \\
Insuficiencia renal aguda & 2 & 2,7 \\
Anemia & 4 & $2,6,7,8$ \\
\hline
\end{tabular}


Tabla III. Características endoscópicas, histológicas y evolución clínica

\begin{tabular}{clll}
\hline Paciente $n^{\circ}$ & Localización & Características histológicas & Evolución clínica \\
\hline 1 & Total esófago & Necrosis de la mucosa/submucosa y trombos vasculares & Asintomático (28 semanas) \\
2 & Tercio distal & No realizada & Falleció (10 días) \\
3 & Total esófago & No realizada & Estenosis \\
4 & Tercio distal & Necrosis de la mucosa/submucosa & Nueva ENA (16 semanas) \\
5 & Tercio medio y distal & Necrosis de la mucosa/submucosa y trombos vasculares & Falleció (16 días) \\
6 & Tercio distal & No realizada & Falleció (12 días) \\
7 & Total esófago & No realizada & Falleció (8 días) \\
8 & Tercio distal & No realizada & Falleció (11 días) \\
9 & Tercio medio y distal & Necrosis de la mucosa/submucosa & Falleció (9 días) \\
10 & Tercio distal & Necrosis de la mucosa/submucosa y trombos vasculares & Falleció (15 días) \\
11 & Tercio medio y distal & Necrosis de la mucosa/submucosa y trombos vasculares & Asintomático (11 semanas) \\
\hline
\end{tabular}

Las biopsias de esófago se obtuvieron en 6 pacientes, el estudio histológico demostró la necrosis de la mucosa y submucosa. El seguimiento medio fue de 11 semanas (1-28 semanas). Durante el seguimiento 4 pacientes mostraron la resolución completa de las lesiones esofágicas. La resolución se observó en otros 2 pacientes, pero en un caso se verificó una estenosis, el otro se presentó 16 semanas después del primer episodio con un nuevo caso de ENA. Siete pacientes fallecieron entre el $8^{\circ}$ e $16^{\circ}$ día de permanencia en el hospital, pero la mayoría de las muertes fueron causadas por la enfermedad de base y no fueron atribuibles a la ENA (Tabla III).

\section{DISCUSIÓN}

La esofagitis necrosante aguda es una patología de descripción muy infrecuente y su frecuencia no está totalmente aclarada. ENA es una muy rara entidad, su frecuencia encontrada en necropsias es del 0,2\%. Segun Etienne y cols. (5) la ENA ocurre en contexto de pacientes con enfermedades sistémicas graves.

Más frecuentemente los pacientes se presentan con una hemorragia digestiva alta. El diagnóstico se estableció por una coloración negra en la mucosa esofágica tras excluir la ingestión de agentes corrosivos, infecciones víricas o fúngicas y pseudomelanosis esofágica (6).

La patogénesis es multifactorial y su etiología no está aclarada. Muchos estudios sugieren el origen isquémico como responsable de la ENA, teniendo como base la clínica y el estudio histológico (7). La asociación a situaciones de bajo flujo y la rápida resolución de algunos casos tras la estabilización hemodinámica sugiere que la reducción temporal del flujo esofágico es responsable de la necrosis esofágica. El compromiso del tercio distal esofágico, es debido a la menor red vascular en esta localización (8).

El malo estado nutricional puede comprometer el sistema de defensa de la mucosa y la capacidad para sanar después de cualquier agresión.

El tratamiento de la ENA no se ha protocolizado pero se deben utilizar bloqueadores de bomba de protones intravenosos y nutrición parenteral.
Se realizaron 4 gastroscopias de control en 4 pacientes que mostraron la resolución de las lesiones esofágicas. Un paciente tuvo un nuevo episodio de ENA 4 meses tras el primer episodio y se recuperó por completo. Dentro de las complicaciones, la principal en nuestro estudio fue una estenosis del esófago que se trató con dilatación. El pronóstico de los pacientes con ENA es malo. Siete pacientes fallecieron, pero las muertes fueron causadas por enfermedades de base y no son atribuibles a la ENA. Dos pacientes están sin síntomas.

En conclusión, la ENA se identificó más veces en nuestro estudio que lo descrito en la literatura, lo que probablemente se deba a la existencia de una población muy envejecida en la región donde se realizó este estudio. En la endoscopia se define por la presencia de una pigmentación negra en el esófago. El tratamiento incluye adecuada hidratación, tratamiento de la enfermedad de base y bloqueadores de la bomba de protones intravenosos. La mortalidad elevada parece deberse a las enfermedades de base y no es atribuible a las lesiones de la ENA. La biopsia probablemente no es necesaria, siempre que exista un cuadro clínico de una pigmentación típica en la endoscopia consistente con ENA.

\section{BIBLIOGRAFÍA}

1. Goldenberg SP, Wain SL, Marignani P. Acute necrotizing esophagitis. Gastroenterology 1990; 98: 493-6.

2. Lacy BE, Toor A, Bensen SP, Rothstein RI, Maheshwari Y. Acute esophageal necrosis: report of two cases and a review of the literature. Gastrointest Endosc 1999; 19: 527-32.

3. Mangan TF, Colley AT, Wytock DH. Antibiotic-associated with acute necrotizing esophagitis. Gastroenterology 1990; 99: 900.

4. Cattan P, Cuillerier E, Cellier C, Carnot F, Landi B, Dusolel A, et al. Black esophagus associated with herpes esophagitis. Gastrointest Endosc 1999; 49: 105-7.

5. Etienne JP, Roge J, Delavierre P, et al. Necroses de l'oesophage d'origine vasculaire. Sem Hop Paris 1969; 45: 1599-606.

6. Gumaste VV, Dave PB. Ingestion of corrosive substances by adults. Am J Gastroenterol 1992; 87: 1-5.

7. Haviv YS, Reinus C, Zimmerman J. "Black esophagus" a rare complication of shock. Am J Gastroenterol 1996; 91: 2432-4.

8. Swigart LL, Siekert RG, Hambley WC, Anson BJ. The esophageal arteries: an anatomic study of 150 specimens. Surg Gynecol Obstet 1950; 90: 234-43. 